\title{
The A. J. Raimondi ISPN Award
}

\section{Concezio Di Rocco}

Published online: 10 June 2009

(C) Springer-Verlag 2009

The A. J. Raimondi ISPN Award was established by the International Society for Pediatric Neurosurgery with the goal of stimulating the publication by young neurosurgeon of innovative basic or clinical studies. It was intended to recognize the spirit of the founder of the journal (Fig. 1) and, in the same time, the scientific curiosity and investigation ability which should characterize the neurosurgeon. Such qualities obvious belonged to the previous

Fig. 1 Prof. Anthony J. Raimondi

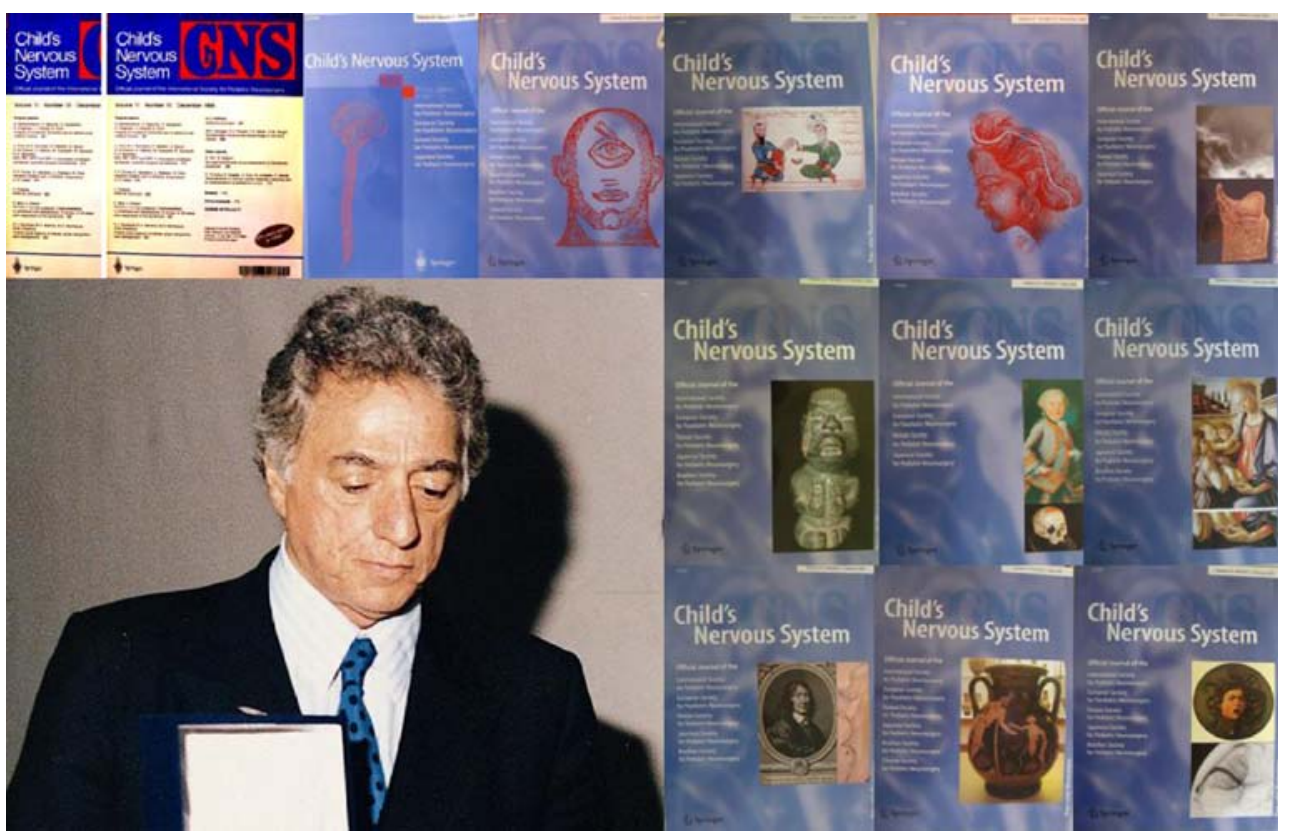

C. Di Rocco $(\bowtie)$

Department of Pediatric Neurosurgery,

Catholic University Medical School,

Largo A. Gemelli 8,

00168 Rome, Italy

e-mail: cdirocco@rm.unicatt.it 


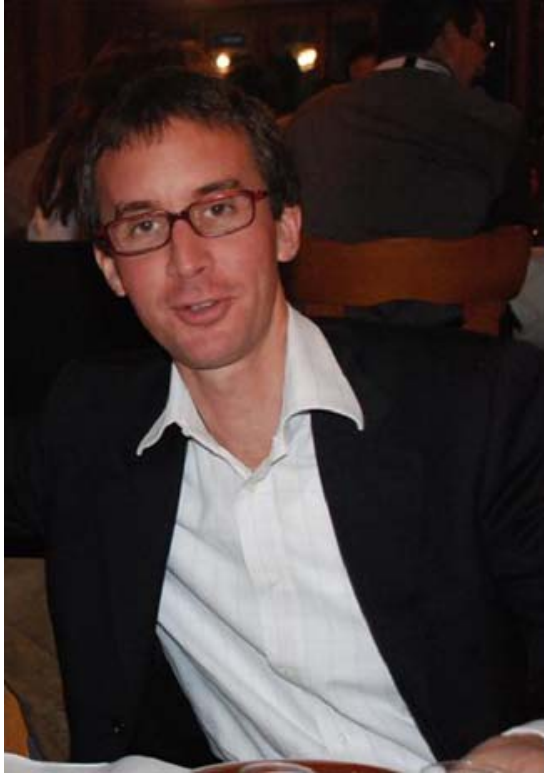

Fig. 2 Dr. Francesco Sala

winners of the award, namely Francesco Sala (Fig. 2; 2002), Vassilios I Vougioukas (Fig. 3; 2003), Yuichiro Nonaka (Fig. 4; 2004), Seok-gu Kang (Fig. 5; 2005), and Daniela Ierardi (Fig. 6; 2007). The same qualities can be observed in the papers by the current winner Anthony Figaji.

Dr. Figaji developed a very active program at the Red Cross Children's Hospital in Capetown to prospectively

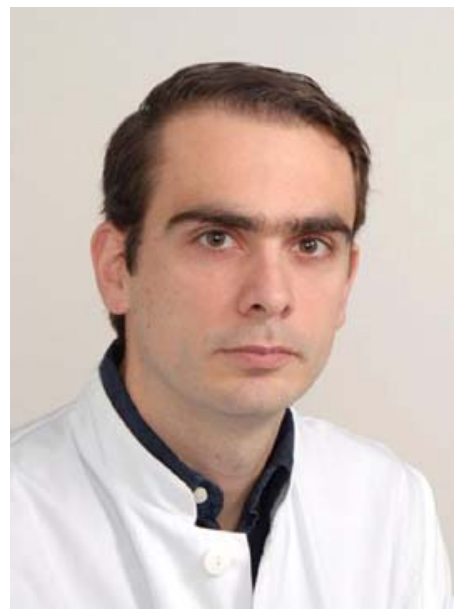

Fig. 3 Dr. Vassilios I Vougioukas

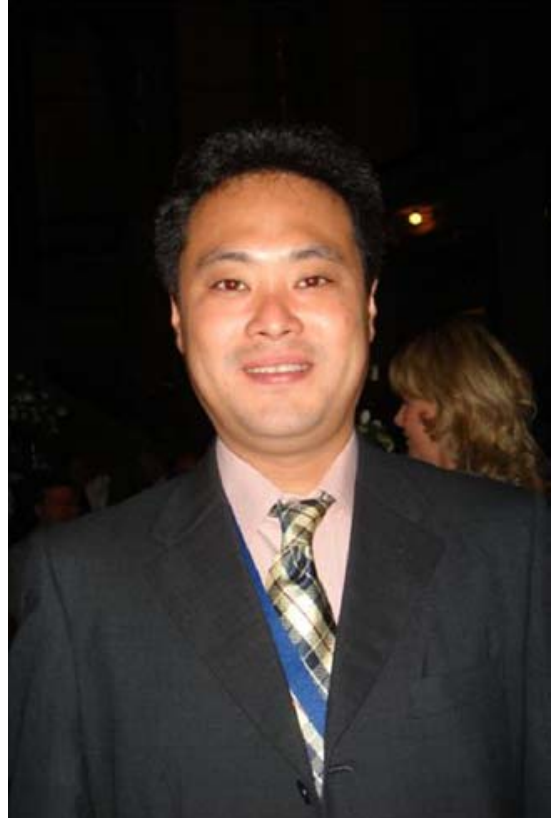

Fig. 4 Dr. Yuichiro Nonaka

evaluate outcome of children suffering from severe traumatic brain injury with particular reference to the impact of multimodalities monitoring on their management. After having demonstrating the importance of this approach in preventing avoidable secondary injury in neurotrauma, the

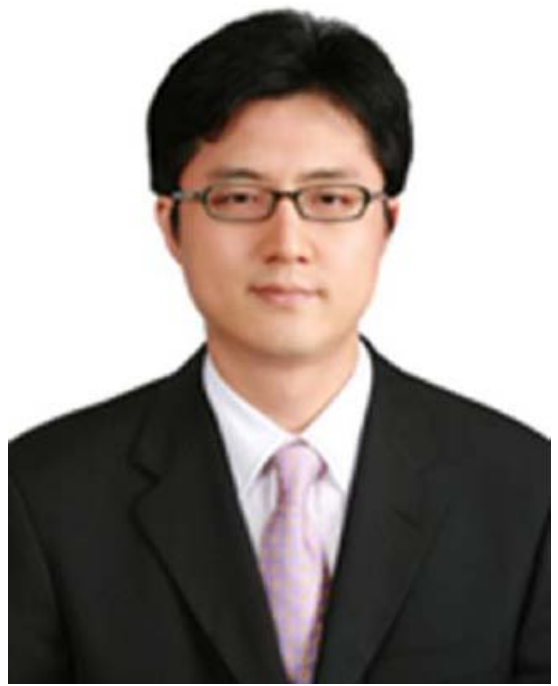

Fig. 5 Dr. Seok-gu Kang 


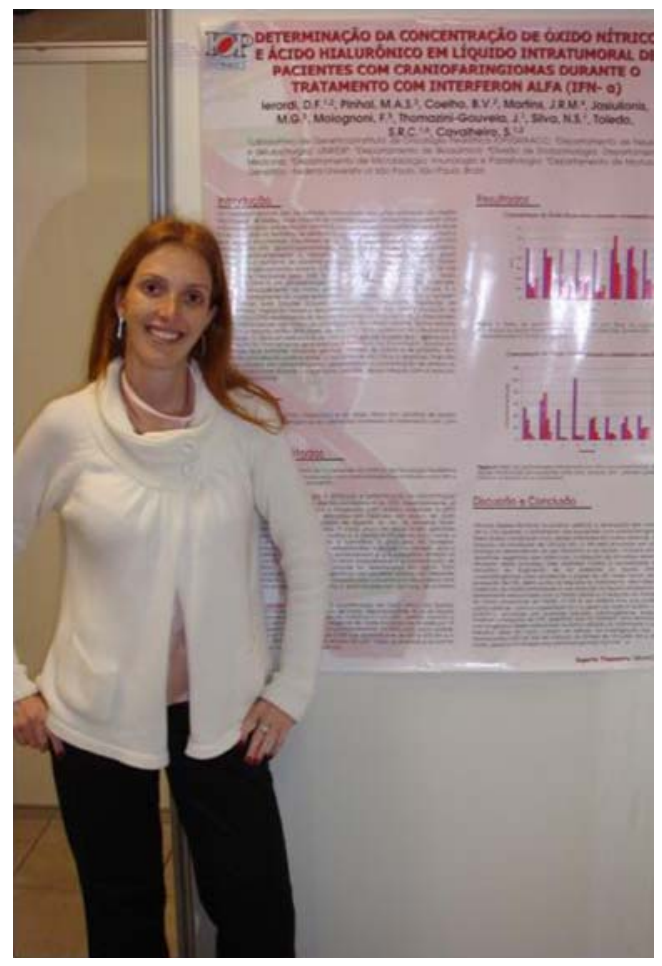

Fig. 6 Dr. Daniela Ierardi program was extended to the management of other pathological conditions sharing with head trauma the potential of additional preventable cerebral injury to the brain, such as subarachnoid hemorrhage, hypoxic-ischemic or infective lesions.

Besides confirming that the management of neurotrauma in children is significantly different from that required for adults patients, the investigations carried out by Dr. Figaji have pointed out the limits of the currently utilized parameters - clinical examination, blood pressure, intracranial pressure - for evaluating the adequacy of brain oxygenation and emphasized the necessity of additional tools, such as brain oxygen and autoregulation monitoring, transcranial Doppler, and biomarkers of injury.

The experience so gained in the last years has allowed Dr. Figaji to establish fruitful cooperation with other clinical centers in North America and Europe aimed at reenergizing the research effort into the management of pediatric brain injury and developing new strategies and protocols for treatment. The papers considered by our journal for the A. J. Raimondi ISPN Award should be regarded as a recognition of this intense research and clinical work and a stimulus for the ongoing projects in the field. 